\title{
Le
}

SECCIÓN: Educación y espacio
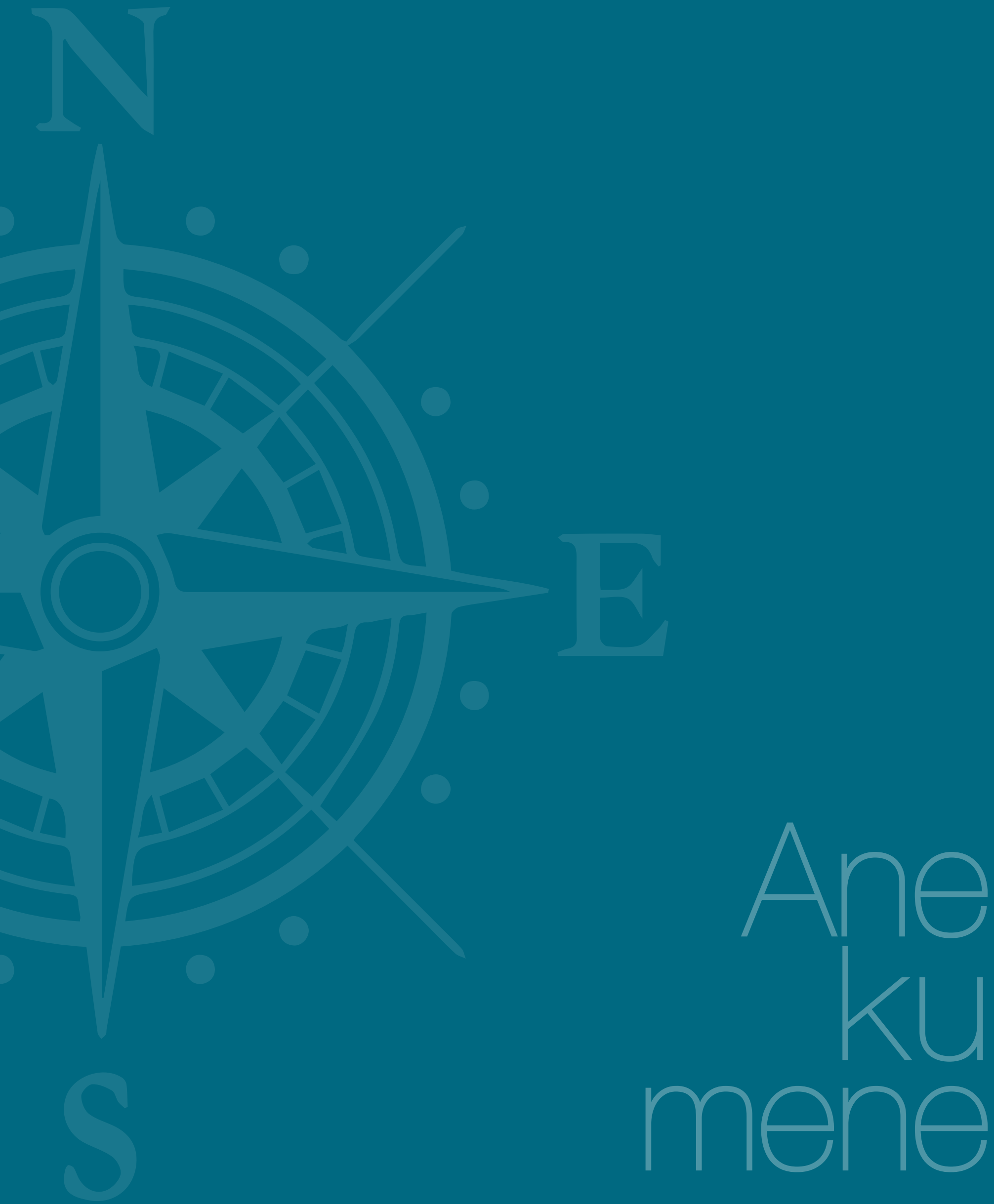


\section{Ciudad y lugar como categorías vividas en el aula}

\section{City and Place as Categories Lived in the Classroom}

\section{Cidade e lugar como categorias vividas na sala de aula}

Juan Fernando Garzón Tejada*

Hernán Darío Díaz Benjumea*

\section{Resumen}

El presente artículo es resultado de un proceso de investigación adelantado en la Institución Educativa Presbítero Antonio José Bernal Londoño, ubicada en la ciudad de Medellín, alrededor de los conceptos de ciudad y lugar en estudiantes de grado sexto. Partiendo de los instrumentos aplicados en la intervención pedagógica, se constata que la experiencia y cotidianidad de los estudiantes configuran representaciones subjetivas de ciudad y, además, favorecen la enseñanza de los conceptos trabajados, puesto que reafirman la importancia del aprendizaje contextual en la educación geográfica y las ciencias sociales.

\section{Palabras clave:}

Entorno escolar, ciudad, lugar, urbano.

\footnotetext{
* Universidad de Antioquia.

** Universidad de Antioquia.
} 


\section{Abstract}

This article is the result of an advanced research process at the Presbitero Antonio José Bernal Londoño Educational Institution, located in the city of Medellín, around the concepts of city and place in sixth grade students. Starting from the instruments applied in the pedagogical intervention, it is verified that the experience and daily life of the students configure subjective representations of the city and, in addition, favor the teaching of the concepts worked, since they reaffirm the importance of contextual learning in geographical education and social sciences.

\section{Resumo}

Este artigo é o resultado de um processo de pesquisa avançada na Instituição Educacional Presbítero Antonio José Bernal Londoño, localizada na cidade de Medellín, em torno dos conceitos de cidade e local em alunos da sexta série. A partir dos instrumentos aplicados na intervenção pedagógica, verifica-se que a experiência e o cotidiano dos alunos configuram representações subjetivas da cidade e, além disso, favorecem o ensino dos conceitos trabalhados, pois reafirmam a importância da aprendizagem contextual na educação geográfica e as ciências sociais.

\section{Keywords:}

School environment, city, place, urban.

\section{Palavras chave:}

Ambiente escolar, cidade lugar, urbano. 


\section{Introducción}

El presente texto se deriva de los resultados del proyecto "Estudio comparado sobre las concepciones de lugar, ciudad, urbano y usos del suelo con profesores y estudiantes de las redes educativas públicas de Sao Paulo-Brasil y Medellín-Colombia". En él se presenta un análisis de los datos obtenidos de los instrumentos aplicados a estudiantes de sexto grado de la Institución Educativa Presbítero Antonio José Bernal Londoño (IEPAJBL), con los que se buscó identificar sus concepciones sobre las categorías objeto de estudio, en el contexto de la enseñanza de la geografía y las ciencias sociales.

La información contenida parte de la investigación realizada en la institución, la cual hace parte del sistema de educación pública de la ciudad de Medellín. Se localiza en la zona noroccidental de la ciudad, Comuna 5, pero dada su cercanía con el sistema de transporte Metro en la Estación Acevedo, su cobertura educativa trasciende y atiende comunidades de los barrios Plaza Colón, La Paralela, Playitas, Héctor Abad, Toscana y otros sectores.

El estudio propició en los estudiantes una experiencia de aprendizaje con referencia a sus espacios habitados, y en los educadores una experiencia pedagógico-investigativa desde el contexto institucional y vinculada a su proceso de enseñanza. El trabajo adelantado plantea para el investigador tres aportes a la educación (aporte al aprendizaje, al saber disciplinar y a la metodología) que se señalan a manera de conclusión al final del texto. El documento se desarrolla atendiendo a las categorías ciudad y lugar exponiendo los elementos emergentes que se tejieron desde los datos obtenidos en los instrumentos aplicados a través del proceso.

\section{Concepciones de ciudad}

La IEPAJBL ${ }^{2}$ está ubicada en un lugar estratégico de la capital antioqueña, lo cual permite la confluencia de estudiantes de diversos sectores de la ciudad y con ello el encuentro de una multiplicidad de estratos económicos, costumbres y culturas que inciden en la concepción de ciudad, lugar, urbano y usos del suelo que poseen los estudiantes. El análisis de los instrumentos aplicados permite rastrear que las vivencias del espacio habitado por parte de los estudiantes proporcionan una idea de esta concurrencia económica, social y cultural, en la medida en que las descripciones encontradas para cada categoría varían de acuerdo con

\footnotetext{
1 En el proceso de investigación participaron por la IE, además de los autores de este texto, los docentes Aleider de Jesús Céspedes Sarrazola, Héctor Jaime Cifuentes Duque, John Byron Alzate Suárez y Marta Inés Pino Pérez.

2 La institución fue fundada en el ańo 2007 como resultado del programa "La educación con calidad es un derecho, no un privilegio", estipulado en el Plan de Desarrollo 2004-2007 por parte de la Alcaldía de Medellín.
}

el lugar donde se habita. Esto se infiere de las descripciones de ciudad, usos del suelo y urbano que hacen los alumnos, registradas a través de las técnicas aplicadas durante el proceso de investigación.

Es importante resaltar que el análisis de las categorías objeto de estudio, llevándolo a términos de Castellar (2011a), posibilita entender el proceso de enseñanza-aprendizaje en educación geográfica como una oportunidad para otorgar sentido a los lugares habitados por los estudiantes. Así, en lo referente a la categoría ciudad, se diseñó un instrumento de tipo narrativo (una carta) con el fin de recolectar datos derivados de la experiencia de vida de los alumnos, buscando reconocer cómo crean y construyen la idea de ciudad que les rodea. En este sentido, se aplicó un instrumento donde se observaron elementos escriturales en primera persona, la carta fue un recurso clave para rastrear las experiencias personales de los estudiantes en relación con la categoría objeto de investigación. Por ejemplo, en la siguiente carta se observa cómo el estudiante hace referencia al espacio geográfico al señalar las montañas que rodean el valle, infraestructuras como museos e iglesias y el sistema de transporte como el metro, todas ellas ideas que se vinculan a la experiencia de ciudad vividas por los sujetos.

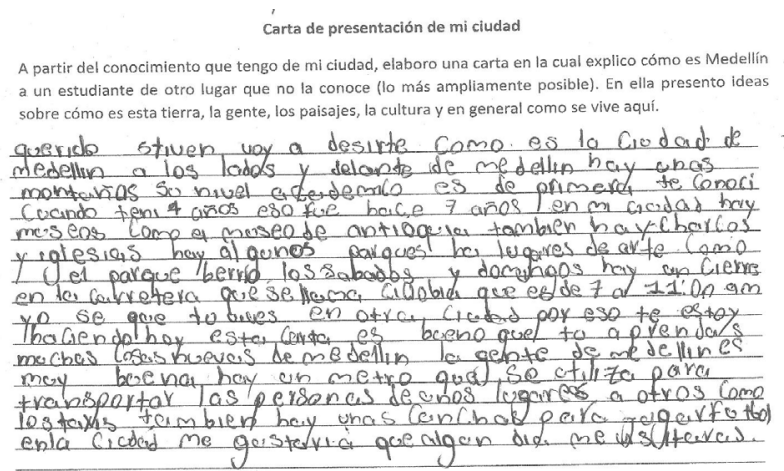

Figura 1. Instrumento 1 aplicado a los estudiantes en el marco de la categoría de ciudad

Escudriñar la concepción de ciudad de los estudiantes desde sus experiencias de vida es una posibilidad para acercar lo que sucede por fuera de las aulas a lo que ocurre en su interior, es desentrañar desde la subjetividad el imaginario que se ha configurado de la ciudad a partir de los encuentros o desencuentros que la misma ha propiciado. Se trata así del reconocimiento de esos saberes que hacen parte de la vida de quienes habitan la ciudad, en nuestro caso de los estudiantes participantes del proyecto, para ponerlos en diálogo con el saber disciplinar que favorece un aprendizaje significativo en la vida escolar. 
Los datos encontrados en las descripciones de las cartas denotan experiencias de ciudad surgidas desde la realidad vivida por cada individuo, en ese sentido, se reflejan perspectivas personales que plantean la manera como se ha vivido la ciudad o como se ha recreado una imagen de la misma desde los medios de comunicación. Se evidencia, igualmente, la influencia de las campañas mediáticas de la Alcaldía de Medellín en la construcción imaginaria de ciudad, puesto que campañas como la de Medellín, Ciudad Innovadora determinaron descripciones ligadas a lo urbano como fuente de oportunidades. Una explicación de este nivel de influencia refiere a la postulación de Medellín como ciudad más innovadora del mundo, por lo que a través de internet, televisión, radio y prensa se convocaba a las personas a votar por la candidatura de la capital.

Este hallazgo permite preguntarse cómo entiende el estudiante una ciudad innovadora y porqué asume esta idea como eslogan personal. En los datos encontrados se puede inferir que la idea de ciudad innovadora se entiende como el tipo de ciudad que, articulada a un sistema de transporte, facilita la movilidad de los ciudadanos. Es una concepción asociada al sistema de transporte público de Medellín que, con el Metro, Metrocable y las rutas alimentadoras, configura una apreciación particular en los participantes. Una explicación a esta concepción de ciudad innovadora es la cercanía de la institución y de las casas de los estudiantes con la Estación Acevedo del Metro, adicional al impacto social que este sistema de transporte ha representado para la capital. De otro lado, como se infiere de las descripciones realizadas, también es una idea externa al estudiante que se alimenta con el paso del tiempo ("es lo que dicen"), por lo que es evidente la influencia de los medios de comunicación en la vida de la población estudiantil.

Los datos obtenidos con el instrumento de la carta permiten inferir que la concepción de ciudad es inherente a la manera en como los jóvenes han vivido la ciudad y de las significaciones que otorga esta experiencia a sus vidas. En este sentido, se encuentran concepciones de ciudad referidas a una ciudad turística, comercial, cultural, educada, bonita y con diversidad de espacios para el esparcimiento y la diversión. Estos elementos se infieren porque el contenido privilegiado se asocia a la infraestructura física de Medellín, es decir, a los espacios públicos de educación (Parque Explora), de esparcimiento (Jardín Botánico, Parque Norte) y de Cultura (Parque de los Deseos, Museo de Antioquia).

En este análisis se hace evidente que el entorno cercano al estudiante provee información de ciudad que se encuentra ante él. No es coincidencia que las descripciones obtenidas se derivan desde una perspectiva contextual, es decir, que responden a los espacios que rodean al sujeto y a los cuales este puede acceder fácilmente. Estudiantes de otra zona de la ciudad posiblemente tengan como referente el Pueblito Paisa, la Unidad Deportiva de Belén y el Aeroparque, puesto que la localización orienta la imagen de ciudad configurada por los seres humanos. La concepción de ciudad subyacente puede responder así a una construcción desde lo cercano y da paso a una configuración social y colectiva que trasciende el ordenamiento legal, los elementos físicos y teóricos para dar lugar a las sensaciones psicosociales que la ciudad confiere. Estamos así ante una idea de ciudad que proporciona una mirada estética que se evidencia cuando se presenta una concepción bonita que entreteje con cierto orgullo el estudiante al resaltar lugares referenciales de Medellín. Es importante resaltar en este punto que la cultura del ciudadano antioqueño posee una idiosincrasia que le proporciona un sentido de pertenencia con la ciudad, lo cual le lleva a sobresaltar y exagerar los logros obtenidos en el pasado. Así, ese querer mostrar lo bueno, lo que el argot popular denomina "chicanear", puede explicar por qué los estudiantes reconocen esos espacios públicos emblemáticos de la ciudad.

No obstante, a pesar de lo anterior, se observa en las cartas recolectadas el reconocimiento de una ciudad que alberga dificultades. Es interesante reconocer en este punto cómo se hallan descripciones favorables a la idea de ciudad, pero no se desconocen las problemáticas que aquejan a sus habitantes y a ellos mismos. Se refleja una ciudad donde acontecen problemas de seguridad, como consecuencia de los hurtos, por las condiciones socioeconómicas de las personas y la vulnerabilidad que implican ciertos lugares o establecimientos. No se esconden las realidades complejas de una ciudad como Medellín y lo más importante es el grado de conciencia de los estudiantes sobre estas situaciones.

La percepción social y de seguridad inferida en los datos plantea un reto particular para la escuela: la formación de ciudadanos que, conscientes de los problemas y las necesidades que aquejan la ciudad, se conviertan en agentes de transformación social para mejorar sus niveles de bienestar y desarrollo. Así, se encuentra aquí una posibilidad de acción en el contexto escolar ya que, si bien el proceso de investigación permite identificar que los estudiantes no son indiferentes a la realidad local, este hallazgo debe orientar la toma de decisiones en los procesos formativos en geografía y ciencias sociales para fortalecer la formación ciudadana con competencias argumentativas y propositivas. Es de resaltar que la Institución Educativa Antonio José Bernal ha reconocido estas posibilidades de formación y, por ello, los estudiantes han participado en procesos de intervención para el afianzamiento de competencias ciudadanas. Esta formación se desarrolla especialmente a través del proyecto Ser Contigo que, mediante una lógica de prevención, acompañamiento y corrección, llevó a la institución a ganar el premio Orden al Mérito Concejo de Medellín, lo cual se traduce en el reconocimiento a las posibilidades de acción desde la escuela para la formación ciudadana.

Por otro lado, siguiendo con el análisis de concepción de ciudad, se encuentra en los datos recolectados la ausencia de una idea con contenido geográfico (físico, económico, humano), ya que no se denotan descripciones vinculadas a las montañas, al valle o al mismo río que hace parte del entorno, o mejor aún, no se detallan en las descripciones conocimientos disciplinares de grados anteriores. Desde esta perspectiva, se 
comparte la idea de Rodríguez, cuando señala que el conocimiento de ciudad "no pasa de ser un listado de elementos físicos del espacio, es decir una idea asociada con casas, edificios, colegios, tiendas, droguerías y supermercados" (2010, p. 31), situación que, como se ha logrado apreciar, se presenta en la construcción de la idea de ciudad aportada por los estudiantes. De esto se desprende que los estudiantes tienen ideas sobre ciudad "pero desconocen su emplazamiento situacional, su organización, funciones, patrones de ordenamiento, uso de suelo, movimiento de personas, mercancías y bienes" (Rodríguez, 2010, p. 31), aunque es preciso reconocer que son ideas constituidas desde la experiencia personal y ligadas al entorno cercano.

La investigación permitió determinar de esta manera unas ideas de ciudad en los participantes, pero, como se evidenció, estas requieren de su fortalecimiento con el desarrollo de elementos conceptuales y disciplinares para enriquecer los conocimientos que la experiencia ha proporcionado. La misma ciudad puede coadyuvar al fortalecimiento de su concepción porque esta "es a la vez un contenido (se aprende sobre la ciudad), un medio o contexto (se aprende en la ciudad), y un agente (se aprende de la ciudad)" (Alderoqui, 2003, p. 160), lo que facilita el aprendizaje y, además, la reflexión personal y colectiva frente a la misma.

\section{Concepciones de lugar}

En relación con la categoría de lugar, se propuso a los estudiantes un instrumento para facilitar el reconocimiento de lugares de Medellín. En una tabla se solicitó registrar el nombre de diferentes lugares y al frente escribir la sensación producida por ellos, para posteriormente representar mediante dibujos los escenarios elegidos. Comparando este instrumento con el aplicado para la categoría anterior, se encuentra recurrente que los lugares señalados responden al entorno cercano de los estudiantes, por lo que la sensación que produce el lugar hace parte de la experiencia vivida por los sujetos. Desde esta perspectiva, el instrumento aplicado para esta segunda categoría, por ejemplo, permite acercarse desde la experiencia de los chicos y chicas a los diferentes lugares habitados por ellos, además, es llevar la vida que se da por fuera de las aulas al ámbito escolar y cerrar la brecha entre las dinámicas externas e internas que impacta a la escuela.

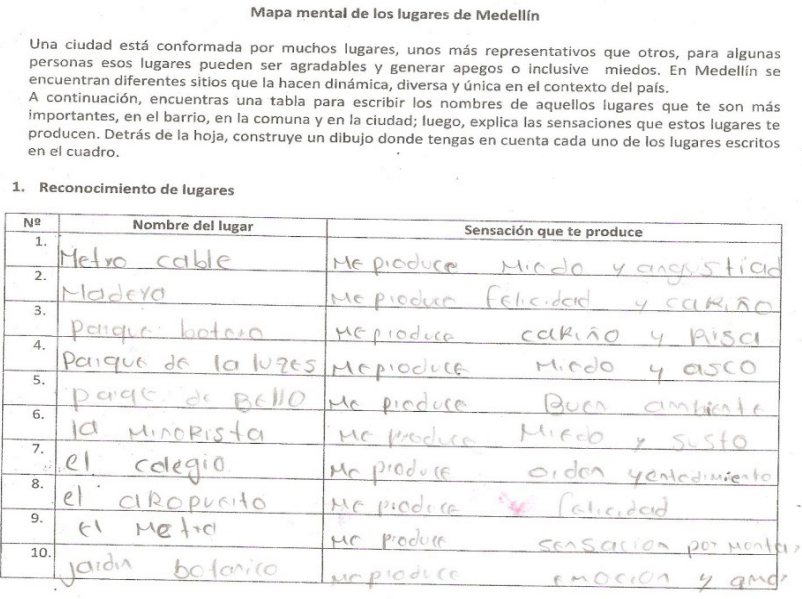

Figura 2. Instrumento 2 aplicado a los estudiantes en el marco de la categoría de lugar

Como se señaló en el apartado anterior, es reiterativo que los medios de transporte son referenciados como lugares que cobran sentido en la vida de los estudiantes, por lo que a ellos se asocian sensaciones de alegría, felicidad y conocimiento. Desde acá se puede llevar a los jóvenes a problematizar sobre la necesidad de transportes en cable dada la geografía de la ciudad y, a su vez, analizar esta necesidad con los miedos o prejuicios de las personas frente a las alturas. Una clase que otorgue sentido a los lugares de los estudiantes, a sus saberes espaciales, puede problematizarse a través de diferentes preguntas que inviten a la reflexión: ¿cómo movilizar a las personas de mi barrio en cable cuando existen miedos frente a su uso?, ¿qué impacto produce en las personas de mi barrio el hecho de tener que movilizarse por cables?, ¿se reconoce en el entorno que los cables son una respuesta de movilidad para nuestra geografía? Se trata, entonces, de tomar la experiencia personal y espacial del estudiante para comprender el espacio enseñado en el aula, y poder de esta manera superar la simple memorización de conceptos tan arraigada tradicionalmente.

De igual manera, abordar el entorno del estudiante desde la ciudad habitada, por ejemplo, puede contribuir al reconocimiento social y cultural de diversos lugares (Castellar, 2011b). Las sensaciones expresadas frente a los lugares señalados en el instrumento por parte de cada estudiante (sentimientos de alegría, tristeza, miedo, tranquilidad, felicidad, entre otros) son expresiones de la experiencia vivida y del arraigo construido alrededor del espacio, lo que facilita la identificación de escenarios tranquilos, deseables y favorables para todos. Es de resaltar que desde este estudio se puede comprender que el reconocimiento social y cultural de un lugar está mediado por la experiencia vivida que se tiene de él. Cultural y socialmente una cárcel puede no representar alegría o felicidad, 
pero puede estar asociada a otro tipo de sentimientos positivos. Así, por ejemplo, para unos estudiantes la iglesia representa apoyo y tranquilidad, quizás transmitidos a través de las prácticas de sus padres, pero para otros significa pereza y tiempo desperdiciado. Este tipo de hallazgos permite reconocer a un sujeto (el estudiante) con historias espaciales específicas que construye su mundo vivido con el paso del tiempo.

Además de lo anterior, es claro que los estudiantes son conocedores de las lógicas de poder y de las situaciones que se viven en sus lugares de residencia. Los jóvenes reconocen la presencia de microtráfico de drogas, de grupos armados que extorsionan y otras realidades que aquejan sus contextos que posiblemente derivan de la ausencia de familiares por homicidio o por reclusión en centro penitenciario. En el instrumento planteado para esta categoría, son diversos los lugares relacionados por parte de los participantes, algunos más recurrentes que otros, pero la generalidad da cuenta de lugares referidos a espacios públicos del área metropolitana. En cada uno de estos escenarios se perciben recuerdos y experiencias albergadas en cada sujeto y son precisamente estos elementos los que construyen la ciudad que vive el estudiante. De esta forma, se presenta una ciudad donde el miedo y el peligro son latentes, especialmente al evocar el Parque de las Luces, el Parque Berrío y la Minorista, pero que asimismo contrasta con la seguridad de lugares como el Parque Arvi, el Jardín Botánico, Parque Norte y el Parque Explora.

Un punto de encuentro con la categoría ciudad es que los estudiantes no se preocupan por mostrar una ciudad bonita y con todas las comodidades que conlleva. Se refleja nuevamente el reconocimiento de las problemáticas que aquejan a la misma, lo cual indica que las generaciones actuales ven en su ciudad un conjunto de lugares que representan bienestar, diversión, pero también situaciones de inseguridad. A la par, otro elemento a resaltar es que los datos recogidos plantean una importancia frente a la relación que los estudiantes tienen con los lugares significativos en sus vidas.

En este sentido, llama la atención en el análisis de los instrumentos tres lugares muy recurrentes: la casa, el colegio y la iglesia. Encontrar la casa como uno de los lugares más importantes para los estudiantes es bastante significativo, más aún cuando a ella se asocian sensaciones de alegría, felicidad, tranquilidad y seguridad. Pero, ¿por qué es tan significativa la casa? Se puede inferir que la familia ocupa un puesto especial en la vida de los estudiantes porque en casa se cuenta con los afectos, el cariño y el cuidado que como personas en crecimiento demandan, y esa especialidad es evidente en los dibujos realizados porque la presencia de la casa se hace visible en la representación realizada. Valdría la pena preguntarse qué sucede con los estudiantes que no incluyeron la casa como uno de sus lugares más importantes. Al hacer el ejercicio, ¿solo pensaron en lugares públicos de la ciudad?, ¿los ambientes familiares de casa no son atractivos para considerarlos como importantes?, ¿se presenta un hogar disfuncional?

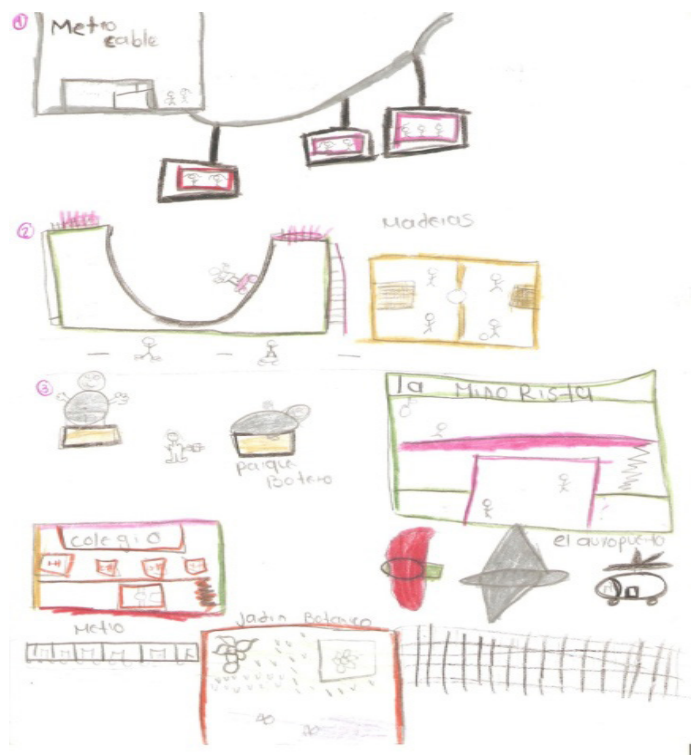

Figura 3. Representación de los lugares realizada por un estudiante

En otro orden de ideas, es menester expresar que en la cotidianidad se suele señalar en muchos jóvenes su pereza frente al estudio y, por tanto, se ha creado frente a ellos un estigma de apatía, indiferencia y desagrado frente a la escuela. Sin embargo, los datos recogidos en la investigación reflejan lo contrario y se presenta el colegio como uno de los lugares más importantes para la vida de los chicos y chicas. Aunque no se puede generalizar, estos datos dan cuenta de un sujeto que encuentra en el colegio un lugar dotado de significado para su vida y de encuentro con sus pares para generar vínculos diferentes al familiar, que se vuelven importantes para el ser humano. Con la iglesia sucede algo similar al colegio: se ha estigmatizado a los jóvenes señalando su indiferencia frente a las prácticas religiosas, pero en este análisis se le identifica como un lugar de experiencia trascendental y espiritual que se convierte en oración, tranquilidad y seguridad. Se detalla en esta perspectiva que para los chicos y chicas la necesidad espiritual y de encontrarse con Dios adquiere relevancia, por lo que este tipo de lugares cobra importancia en sus vidas y la de sus familias.

Según el análisis, los dibujos de esta categoría son congruentes con los lugares señalados en la tabla. Un elemento significativo en los dibujos es la presencia de objetos específicos de los lugares indicados por los estudiantes. De esta forma, para la representación de un lugar como Plaza Botero se hace uso de las figuras de este artista, para el Metrocable las respectivas cabinas de transporte, y para el Parque Norte los juegos mecánicos que generan mayor adrenalina. 


\section{A modo de conclusión}

Los docentes co-investigadores se interesaron por comprender las concepciones de ciudad y lugar de los estudiantes de sexto de la IE Antonio José Bernal Londoño. El tratar las categorías objeto de estudio desde la enseñanza de la geografía y las ciencias sociales ha permitido propiciar una experiencia de enseñanza-aprendizaje que otorga importancia a los espacios habitados por los estudiantes y los cuales adquieren significado al vincularlos con lo que se hace en las clases. En relación con el análisis de las categorías, se observa en la de lugar una concepción que se vincula con el entorno y el espacio habitado por los estudiantes. De ahí que este se convierte en una forma de afianzar la identidad con el espacio habitado, en la medida en que los jóvenes se apropian de un espacio y crean un significado desde la experiencia personal obtenida. Asimismo, en la categoría ciudad se denota una concepción de espacio que otorga sentido a la vida. Las experiencias de los estudiantes reflejan unas perspectivas personales que expresan cómo han vivido los espacios de ciudad. De esta manera, estos espacios confieren percepciones de aprendizaje, alegría, diversión, miedo, entre otros, que van dando sentido a la ciudad que se habita.

El estudio permitió a los coinvestigadores propiciar en los estudiantes una experiencia de aprendizaje con referencia a sus espacios habitados, y en los educadores una experiencia pedagógico-investigativa desde el contexto institucional y vinculada a su proceso de enseñanza. El proceso realizado plantea para el investigador tres aportes a la educación: al saber disciplinar, al aprendizaje y a la metodología. En primer lugar, es una contribución al reconocimiento del saber disciplinar de los estudiantes valorar sus concepciones y por tanto sus saberes frente a los conceptos de ciudad y lugar. De esta forma, el proceso de enseñanza en educación geográfica y en ciencias sociales parte de los conocimientos previos de los estudiantes, su experiencia personal y el entorno en el cual se desenvuelven, haciendo posible establecer horizontes de formación en las categorías trabajadas para permitir la configuración de un saber conceptual y disciplinar. En segundo lugar, la investigación adelantada es un aporte al saber disciplinar del docente, entendido este como el campo donde subyacen un conjunto de conocimientos (categorías, conceptos, nociones) derivados de la producción científica y de los cuales un sujeto toma posesión para llevarlos a su enseñanza en un contexto educativo. Esto obedece a que los docentes participantes en el proceso cualifican su saber en la medida en que aprenden de su disciplina de la mano de la investigación.

Asimismo, se encuentra un aporte al proceso metodológico en la enseñanza de la educación geográfica y en ciencias sociales al propiciar procesos orientados desde el entorno y contexto del estudiante. Para materializar este aporte metodológico en el aula, los docentes planean y diseñan unidades didácticas orientadas a la enseñanza de ciudad y lugar porque, como manifiesta Rodríguez (2010), se hacen necesarias para la enseñanza de la geografía o de cualquier otra asignatura. Estas unidades parten de las concepciones encontradas y desde allí se establece la ruta de formación más idónea para favorecer el aprendizaje de las categorías manejadas y su comprensión. Este tipo de propuestas inciden favorablemente en la enseñanza de la geografía y de las ciencias sociales al permitir una enseñanza basada en los contextos de los estudiantes, proporcionando de esta forma sentido a lo que se aprende y alejándose de una enseñanza tradicional que muchas veces se limita a "copiar en el cuaderno las informaciones o resúmenes que se tienen que memorizar" (Rodríguez, 2010, p. 30).

Finalmente, se logra evidenciar un aporte al aprendizaje al permitir dar significado a los lugares habitados por los estudiantes, relacionando los saberes formales e informales y permitiendo articular los conocimientos científicos a las vivencias de los sujetos. El estudio muestra que el entorno y la experiencia sobre este otorgan unos saberes que la escuela puede usar para analizar desde la cotidianidad los fenómenos que se presentan en el espacio. Es la posibilidad de propiciar un conocimiento situado "que propone contextualizar los contenidos, relacionándolos con la realidad que vive el estudiante, lo que produciría aprendizajes significativos" (Rodríguez, 2010, p. 61).

\section{Referencias}

Alderoqui, S. (2003). La ciudad: un territorio que educa. Caderno CRH, (38), 153-176.

Castellar, S. (2011a). A cidade como método de estudo na educação geográfica. En A. Cely y N. Morenos (ed.), Ciudades leídas, ciudades contadas. La ciudad como escenario didáctico para la enseñanza de la geografía (pp. 153-170). Bogotá: Universidad Distrital Francisco José de Caldas.

Castellar, S. (2011b). La superación de los límites para una educación geográfica significativa: un estudio sobre la ciudad y en la ciudad. Anekumene, (1), 67-84.

Rodríguez, E. (2010). Geografía conceptual. Enseñanza y aprendizaje de la geografía en la educación básica secundaria. Bogotá: Tercer Mundo. 